\title{
A!
}

This is an electronic reprint of the original article.

This reprint may differ from the original in pagination and typographic detail.

Manninen, Matti; Nørskov, J. K.; Puska, M. J.; Umrigar, Cyrus

\section{Repulsive interaction of the helium atom with a metal surface}

Published in:

Physical Review B

DOI:

10.1103/PhysRevB.29.2314

Published: 15/02/1984

Document Version

Publisher's PDF, also known as Version of record

Please cite the original version:

Manninen, M., Nørskov, J. K., Puska, M. J., \& Umrigar, C. (1984). Repulsive interaction of the helium atom with a metal surface. Physical Review B, 29(4), 2314-2316. https://doi.org/10.1103/PhysRevB.29.2314

This material is protected by copyright and other intellectual property rights, and duplication or sale of all or part of any of the repository collections is not permitted, except that material may be duplicated by you for your research use or educational purposes in electronic or print form. You must obtain permission for any other use. Electronic or print copies may not be offered, whether for sale or otherwise to anyone who is not an authorised user. 


\title{
Repulsive interaction of the helium atom with a metal surface
}

\author{
M. Manninen \\ Research Institute for Theoretical Physics, University of Helsinki, \\ SF-00170 Helsinki 17, Finland \\ J. K. Nørskov \\ Nordisk Institut for Teoretisk Atomfysik (NORDITA), Blegdamsvej 17, \\ $D K-2100$ Copenhagen $\emptyset$, Denmark \\ M. J. Puska \\ Laboratory of Physics, Helsinki University of Technology, \\ SF-02150 Espoo 15, Finland \\ Cyrus Umrigar \\ Laboratory of Atomic and Solid State Physics, Cornell University, \\ Ithaca, New York 14853 \\ (Received 25 October 1983)
}

\begin{abstract}
The repulsive part of the helium scattering potential at a surface is approximately proportional to the surface electron density. The proportionality coefficient is shown to be a well-defined quantity, which can be related to the electron-helium scattering length. The spread in the values of the proportionality constant suggested in the literature is shown to be due to different definitions of the coefficient or due to inadequate calculational methods. The value calculated using the local density approximation with a selfinteraction correction is in very good agreement with the electron-scattering-length measurements.
\end{abstract}

Using the effective-medium theory, ${ }^{1,2}$ Esbjerg and Norskov ${ }^{3}$ showed that the leading repulsive term in the helium scattering potential from any electronic system is proportional to the local unperturbed electron density $n_{0}(\vec{r})$ of the host at the helium site:

$$
V_{R}(\vec{r})=\alpha n_{0}(\vec{r}) \text {. }
$$

A more refined treatment ${ }^{4}$ gives

$$
V_{R}(\overrightarrow{\mathrm{r}})=\alpha_{\mathrm{eff}} \bar{n}_{0}(\overrightarrow{\mathrm{r}}) \text {, }
$$

where $\bar{n}_{0}$ is the average of $n_{0}(\vec{r})$ over the electrostatic potential $\Phi_{\mathrm{He}}(\vec{r})$ of the helium atom, and

$$
\alpha_{\text {eff }}=\alpha-\int d^{3} r \Phi_{\mathrm{He}}(\overrightarrow{\mathrm{r}})
$$

is a new effective proportionality constant. Unfortunately, the value of $\alpha$ published in the original paper ${ }^{3}$ was wrong due to numerical errors. This together with the occurrence of two constants $\alpha$ and $\alpha_{\text {eff }}$ and other approaches ${ }^{5,6}$ for determining the coefficient has resulted in several values for $\alpha$ and has given an impression that theoretically $\alpha$ is not well defined or that the correct value is not known. This confusion is also increased by the fact that, keeping $\alpha$ as a free parameter, good agreement with the experimental results has been achieved for helium diffraction from metal surfaces. ${ }^{7}$

The purpose of the present Rapid Communication is to comment upon the differences between various values of the proportionality constant. We point out that $\alpha_{\text {eff }}$ is related to the scattering length for slow electrons on atomic helium. It can thus be determined experimentally. The $\alpha_{\text {eff }}$ used in the effective-medium theory is in good agreement with the experimentally determined value.

The density functional theory ${ }^{8}$ expresses the ground-state energy as a functional of the total electron density of the system. In the effective-medium theory one goes a step further and shows that the embedding energy of the helium atom in any electronic surrounding can be calculated as a functional of the unperturbed electron density of the host. Formally, one can always separate a local term from this functional and write

$$
V_{R}(\overrightarrow{\mathrm{r}})=\Delta E\left(n_{0}(\overrightarrow{\mathrm{r}})\right)+\Delta E_{\mathrm{nl}},
$$

where $\Delta E\left(n_{0}\right)$ is the embedding energy of helium in a homogeneous electron gas of density $n_{0}, n_{0}(\vec{r})$ is the unperturbed surface electron density, and $\Delta E_{\mathrm{nl}}$ includes all nonlocal terms. $\Delta E\left(n_{0}\right)$ is calculated by embedding the helium atom in a homogeneous electron gas with a compensating positive background charge. ${ }^{9}$ In the low-density limit this gives $\Delta E(n)$ proportional to the density and thereby Eq. (1). We have calculated $\Delta E(n)$ for low electron densities using the local density approximation ${ }^{10}$ (LDA) for the exchange and correlation energies with and without a selfinteraction correction ${ }^{11}$ (SIC). The corresponding slopes $\alpha$ are given in Table $I$.

In the region outside a metal surface sampled by a lowenergy $(<500 \mathrm{meV})$ helium atom there are no positive charges. Also, the surface electron density has nonnegligible gradients. In the refined treatment, Eqs. (2) and (3), the former problem is taken into account by subtracting from Eq. (1) the electrostatic interaction of the helium atom with the homogeneous background [Eq. (3)]. This results in the new proportionality constant $\alpha_{\text {eff }}$ in Eq. (2), which is also given in Table $I{ }^{15}$ The use of an averaged surface electron density $\bar{n}_{0}(\vec{r})$ in Eq. (2) is the lowest-order inclusion of nonlocality. Outside a metal surface we typically find $\bar{n}_{0}(\vec{r}) \simeq 1.3 n_{0}(\vec{r})$ fairly independent of $\vec{r}$ for the range of positions of relevance for low-energy helium scattering. This means that we can define a third proportionality constant

$$
\bar{\alpha}_{\text {eff }}=\left[\bar{n}_{0}(\vec{r}) / n_{0}(\vec{r})\right] \alpha_{\text {eff }}
$$


TABLE I. Proportionality constants for the helium-metal scattering potential.

\begin{tabular}{lccc}
\hline \hline & $\alpha\left(\mathrm{eV} a_{0}^{3}\right)$ & $\alpha_{\mathrm{eff}}\left(\mathrm{eV} a_{0}^{3}\right)$ & $\bar{\alpha}_{\mathrm{eff}}\left(\mathrm{eV} a_{0}^{3}\right)$ \\
\hline Effective-medium approach & & & \\
$\quad$ LDA & 305 & 160 & 208 \\
$\quad$ LDA-SIC & 329 & 196 & 1000 \\
Harris-Liebsch approach & & 517 & -500 \\
$\quad$ First-order (Ref. 5) & & 203 & \\
Second-order (Ref. 12) & $191, \ldots, 203$ & \\
Scattering length & & \\
$\quad$ Calculation (Ref. 13) & & \\
Experimental (Ref. 14) & & \\
\hline \hline
\end{tabular}

so that

$$
V_{R}(\overrightarrow{\mathrm{r}})=\bar{\alpha}_{\text {eff }} n_{0}(\overrightarrow{\mathrm{r}}) .
$$

$\bar{\alpha}_{\text {eff }}$ is also included in Table I. It should be stressed that $\bar{\alpha}_{\text {eff }} / \alpha_{\text {eff }}$ depends on the kind of system considered and that $\bar{\alpha}_{\text {eff }}$ in Table I is only applicable for helium-metal surface interactions over a limited range of distances.

Following Zaremba and Kohn, ${ }^{16}$ Harris and Liebsch ${ }^{5}$ have shown that, to first order in the overlap between the surface and helium states, the repulsive part of the helium-surface potential can be written

$$
V_{R}(\overrightarrow{\mathrm{r}})=\int_{-\infty}^{\epsilon_{F}} d \epsilon g(\epsilon) n_{0}(\epsilon, \overrightarrow{\mathrm{r}}),
$$

where $g(\epsilon)$ is a universal function of energy only, and $n_{0}(\epsilon, \vec{r})$ is the local density-of-states function of the bare surface. Formally Eq. (6) can be written

$$
V_{R}(\overrightarrow{\mathrm{r}})=g(\bar{\epsilon}) n_{0}(\overrightarrow{\mathrm{r}}),
$$

where $\overline{\boldsymbol{\epsilon}}$ in principle depends on $\overrightarrow{\mathbf{r}}$. We have made explicit calculations within the local density approximation, using the linear augmented plane-wave method for a five-layer $\mathrm{Ni}(110)$ slab, ${ }^{17}$ which show that in reality $\bar{\epsilon}$ is basically constant for the range of distance of interest here $\left[n_{0}(\epsilon, \vec{r})\right.$ is dominated by energies close to the Fermi energy]. This has also been argued by Harris and Liebsch ${ }^{5}$ based on jelliumsurface calculations. The Harris-Liebsch value for $\bar{\alpha}_{\text {eff }}$ is thus $g(\bar{\epsilon})$ and again $\bar{\alpha}_{\text {eff }}$ will depend on the kind of system studied. For a low-density homogeneous electron gas the Harris-Liebsch approach gives $\alpha_{\text {eff }}=g(0)$. The HarrisLiebsch values for $\bar{\alpha}_{\text {eff }}$ and $\alpha_{\text {eff }}$ calculated within the Hartree-Fock approximation are shown in Table I. In the table we include both the originally published values (first order in the helium pseudopotential) and the most recent ones by Nordlander and Harris. ${ }^{12}$ The latter are calculated to second order in the helium pseudopotential and the energy argument in $g(\epsilon)$ is replaced by $\epsilon-v_{0}(\vec{r})$, where $v_{0}(\vec{r})$ is the host one-electron potential at the helium position. ${ }^{12}$ The Harris-Liebsch approach is not designed for the problem of helium in a uniform electron gas, and the perturbation theory which involves a polarizability does not converge well $^{18}$ as the electron gas density and bandwidth go to zero. We therefore have not included any second-order value for $\alpha_{\text {eff }}$ in Table I (treated in all orders of the perturbation theory the Harris-Liebsch approach would lead to the exact value extracted from the scattering length; see below). It is seen how the value $\bar{\alpha}_{\text {eff }}$ differs dramatically from the effective medium value, which is rather independent of the approximation used for the treatment of exchange and correlation effects. Most of this difference is found again in $\alpha_{\text {eff }}$.

Now we want to point out that there is a more direct way

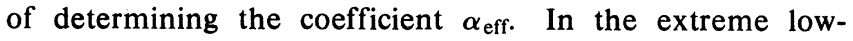
density limit the interaction of electrons with a helium atom can be calculated from scattering theory. For $N$ electrons in a volume $V$ (without the positive background) the embedding of a helium atom increases the total energy of the electrons by

$$
\Delta E=\frac{4 \pi \hbar^{2}}{2 m} a_{s} \frac{N}{V},
$$

where $a_{s}$ is the scattering length. A comparison with Eq. (1) gives directly

$$
\alpha_{\mathrm{eff}}=\frac{2 \pi \hbar^{2}}{m} a_{s}
$$

This approximation for the electron-helium interaction has conventionally been used in studying the electron-induced bubbles in liquid helium (see Ref. 19 and references therein). The advantage is that the scattering length can be computed by solving a three-electron problem and a more elaborate many-body theory can be applied. The $\alpha_{\text {eff }}$ values determined from theoretical and experimental scattering lengths are included in Table I. A comparison shows that the LDA gives good results and LDA-SIC give excellent results for the helium interaction with the low-density electron gas. The results of Table I do, however, indicate some problems with the Harris-Liebsch value for $\alpha_{\text {eff. The }}$ The two possible explanations of the too-large value. It may be that the expansion in the helium-metal overlap or the perturbation theory in the helium pseudopotential is not converged. The other possibility is the difference in the treatment of exchange and correlation effects between the Hartree-Fock (HF) and LD approximations used in the two approaches. In the HF approximation of the Harris-Liebsch approach, ${ }^{5,12}$ no correlation effects are included, whereas in the LD approximation and, in particular, when the selfinteraction correction is included, correlation effects are treated to a certain approximation. The LDA value of $\bar{\alpha}_{\text {eff }}$ is therefore less repulsive than the Hartree-Fock value. If the too-large value of $\alpha_{\text {eff }}$ in the Harris-Liebsch-Nordlander treatment is due to a lack of correlation effects in the HF treatment, this need not invalidate the approach for calculat- 
ing the He-surface interaction. All correlation effects are in principle included in the zeroth order contribution to the interaction potential in the overlap expansion which is the van der Waals attraction. In praxis the asymptotic form of the van der Waals interaction is added to the HF repulsive potential Eq. (6). Such a procedure gives, for instance, a reasonable description of the physisorption minimum when compared to experiment. In the effective-medium approach, on the other hand, the exchange-correlation energy of the helium atom in a homogeneous electron gas is already included in the repulsive part $V_{R}$ of the potential, and only the extra exchange-correlation energy due to the inhomogeneity of the surface electron density must be added. Model calculations for jellium surfaces show that this is a small effect which is important only far from the surface, where $V_{R}$ is small. With the inclusion of the extra exchange-correlation energy the effective-medium approach provides a good description of the helium-jellium-surface potential including the physisorption minimum, when compared to a fully self-consistent calculation within the local density approximation. 4,20

Providing the overlap expansion is converged, the choice of the value for the proportionality constant $\bar{\alpha}_{\text {eff }}$ in the repulsive part of the helium-surface potential thus depends on the choice of the treatment of the exchange and correlation effects. If the asymptotic form of the van der Waals interaction is included, the corresponding large (HF) value of $\bar{\alpha}_{\text {eff }}$ must be used. This description is clearly the most appropriate far from the surface. Closer in, care must be taken with the divergence of the asymptotic van der Waals form. ${ }^{12}$ If a local density description of the exchange and correlation is chosen, the small value of $\bar{\alpha}_{\text {eff }}$ should be used and only the extra exchange-correlation energy due to the inhomogeneity of the surface electron density added. This correction is small. The local density description is expected to work better the closer the He atom is to the surface.

\section{ACKNOWLEDGMENTS}

Discussions with J. Harris, N. Lang, R. M. Nieminen, and P. Nordlander are gratefully acknowledged. We are grateful to P. Nordlander for providing us with his results before publication. One of us (C.U.) is grateful to the Office of Naval Research for financial support.
${ }^{1}$ J. K. Nørskov and N. D. Lang, Phys. Rev. B 21, 2131 (1980).

${ }^{2}$ M. J. Stott and E. Zaremba, Phys. Rev. B 22, 1564 (1980).

${ }^{3}$ N. Esbjerg and J. K. Nørskov, Phys. Rev. Lett. 45, 807 (1980).

${ }^{4}$ N. D. Lang and J. K. Nørskov, Phys. Rev. B 27, 4612 (1983).

${ }^{5}$ J. Harris and A. Liebsch, J. Phys. C 15, 2275 (1982).

${ }^{6}$ R. B. Laughlin, Phys. Rev. B 25,2222 (1982).

${ }^{7}$ R. Schinke and A. C. Luntz, Surf. Sci. Lett. 124, L60 (1983),

${ }^{8}$ P. Hohenberg and W. Kohn, Phys. Rev. 136, B864 (1964); W. Kohn and L. J. Sham, ibid. 140, A1133 (1965)

${ }^{9}$ M. J. Puska, R. M. Nieminen, and M. Manninen, Phys. Rev. B $\underline{24}$, 3037 (1981).

${ }^{10}$ O. Gunnarsson and B. I. Lundqvist, Phys. Rev. B 13, 4274 (1976).

${ }^{11} \mathrm{~J}$. Perdew and A. Zunger, Phys. Rev. B 233, 5048 (1981).

${ }^{12} \mathrm{P}$. Nordlander and J. Harris (unpublished).

${ }^{13}$ N. R. Kestner, J. Jortner, M. H. Cohen, and S. A. Rice, Phys. Rev. 140, A56 (1965).

${ }^{14}$ T. F. O'Malley, Phys. Rev. 130,1020 (1963).

${ }^{15}$ In principle, the integral in Eq. (3) and in the definition of $\bar{n}_{0}$ is only over the region close to the $\mathrm{He}$ atom where the atomic potential dominates (Ref. 4). In this region the He-induced electron density in a homogeneous electron gas is approximated very well by that of the free atom. Furthermore, for this He density, $V_{R}$ of Eq. (2) is basically independent of the choice of the radius $R_{c}$ of the near-atom region for $R_{c}>2.5 a_{0}$, even though $\alpha_{\text {eff }}$ and $\bar{n}_{0}(\vec{r}) / n_{0}(\vec{r})$ do depend slightly on $R_{c}$ (Ref. 4). Here we have used $R_{c}=\infty$.

${ }^{16}$ E. Zaremba and W. Kohn, Phys. Rev. B 15, 1769 (1977).

${ }^{17}$ M. Manninen, J. K. Nørskov, and C. Umrigar (unpublished).

${ }^{18} \mathrm{P}$. Nordlander (private communication).

${ }^{19}$ R. M. Nieminen, I. Välimaa, M. Manninen, and P. Hautojärvi, Phys. Rev. A 211, 1677 (1980)

${ }^{20}$ The second-order nonlocal term in the effective-medium theory involves the polarizability of the helium atom in a homogeneous electron gas. This term has been calculated for helium outside a metal surface and it is very small, indicating that the effectivemedium theory is converged with the first-order nonlocal term. 